\title{
A investigação em genômica pediátrica no Departamento de Pediatria da Faculdade de Medicina da Universidade de São Paulo - FMUSP
}

\author{
Pediatric genomics investigation at FMUSP \\ Department of Pediatrics
}

\author{
Carlos Alberto Moreira-Filho
}

\begin{abstract}
Moreira-Filho CA. A investigação genômica pediátrica no Departamento de Pediatria da Faculdade de Medicina da Universidade de São Paulo - FMUSP. Rev Med (São Paulo). 2010 abr.-jun.;89(2):83-7.
\end{abstract}

RESUMO: A susceptibilidade para desenvolver doenças crônicas na vida adulta, tais como diabetes, doenças cardiovasculares ou distúrbios de comportamento, depende em larga medida da interação entre fatores genéticos e ambientais na vida fetal e nos primeiros anos de vida. A investigação de como alterações nos ambientes pré e pós-natal modificam o funcionamento dos genes, promovendo mudanças permanentes no metabolismo e determinando a susceptibilidade a diferentes doenças, constitui hoje o alvo principal da genômica pediátrica. $O$ conhecimento obtido nessa área da Medicina poderá permitir, futuramente, que intervenções específicas em fases iniciais da vida conduzam à melhoria da saúde humana. Este artigo resume avanços recentes em genômica pediátrica e as atividades do Departamento de Pediatria da FMUSP nessa área.

DESCRITORES: Pediatria; Genômica.

\section{INTRODUÇÃO}

O bservações epidemiológicas e estudos em modelos animais têm evidenciado que o risco de desenvolver doenças crônicas na vida adulta, tais como diabetes, doenças cardiovasculares ou distúrbios de comportamento, depende da interação de fatores genéticos e ambientais na vida fetal e nos primeiros anos de vida ${ }^{1}$. Uma nova área de investigação em Medicina, conhecida pela sigla $\mathrm{DOHaD}$, do inglês "Developmental Origins of Health and Disease", está se consolidando, com importantes implicações biológicas, médicas e socioeconômicas². No entanto, ainda são pouco compreendidos os mecanismos pelos quais determinadas alterações nos ambientes pré e pós-natal influenciam o funcionamento dos genes trazendo mudanças permanentes no metabolismo que determinam a susceptibilidade

Professor Associado do Departamento de Pediatria da FMUSP. Laboratório de Investigação Médica em Pediatria Clínica (LIM-36). Instituto da Criança do HC-FMUSP.

Endereço para correspondência: Carlos Alberto Moreira-Filho. Av. Dr. Enéas Carvalho Aguiar, 647 - 5ำ Andar. 05403900, São Paulo, SP, Brasil. e-mail: carlos.moreira@icr.usp.br 
a diferentes doenças. Há necessidade de uma nova abordagem que identifique as associações causais entre fatores ambientais pré e pós-natais, modificações de longo prazo na regulação epigenética (influência de fatores dos meios interno e externo sobre o funcionamento dos genes) e doenças, o que permitirá intervenções específicas em fases iniciais da vida que conduzam à melhoria da saúde humana ${ }^{3}$.

Nesse contexto, é preciso considerar que recentes avanços no conhecimento do genoma humano e a convergência tecnológica envolvendo biologia molecular, imagem, informática e nanotecnologia estão levando a uma mudança de paradigma na Medicina ${ }^{4}$. Terapias passam a ser baseadas no mecanismo molecular da doença e nas características genéticas do paciente. A intervenção, em muitos casos, ocorre antes - e não após - a constatação da doença. A resposta a agentes terapêuticos pode ser monitorada através do estudo da expressão gênica no tecidoalvo. A susceptibilidade a doenças complexas pode ser identificada em diferentes populações através de marcadores genéticos ${ }^{5}$ e estudos prospectivos de coortes em comunidades selecionadas ${ }^{6}$.

Esse cenário teve um impacto importante na área de Pediatria. Em 2001, foi criada nos EUA a primeira cadeira de Genômica Pediátrica na Universidade da Pennsylvania e Children's Hospital of Philadelphia. Nesse mesmo ano, o Centers for Disease Control and Prevention (CDC) estabeleceu um centro para genômica e saúde pública. Em 2002, o CDC iniciou o programa NANHES III para estudo da variabilidade genômica significativa para saúde pública, incluindo banco de dados populacionais e repositório de amostras biológicas. Em 2003, o simpósio do CDC sobre genômica e saúde pública abriu o caminho para o envolvimento das principais escolas médicas americanas em estudos de genética pediátrica e epidemiológica. Em 2004, o CDC lançou o projeto Evaluation of Genomic Applications in Practice and Prevention (EGAPP), para facilitar a introdução de testes genéticos na prática clínica. Em 2005, o National Office of Public Health Genomics (NOPHG) do CDC abrigou o Programa Internacional de Biobancos e Estudos de Coortes e o CDC concedeu financiamento às Universidades de Michigan e Washington para que essas instituições coordenassem o programa "Centers for Genomics and Public Health" com objetivo de integração da genômica à saúde pública e à prática clínica nos EUA. Em 2006, o NOPHG iniciou pesquisa multicêntrica sobre genômica de doenças respiratórias e influenza. Em 2007-2008, foram lançados programas nacionais de pesquisa sobre genômica de doenças crônicas, defeitos congênitos e exposição ambiental. Os portais www.cdc.gov/genomics e www.hugenavigator.net/ trazem informações atualizadas sobre os projetos e iniciativas acima mencionados.

A prevenção das doenças crônicas da vida adulta está se tornando um dos principais desafios da Pediatria no século XXI. Com essa percepção, em 2005, o Departamento de Pediatria da FMUSP elaborou um programa de pesquisa denominado "Uma Nova Puericultura para Crianças que vão viver 100 Anos ou Mais", visando ao desenvolvimento de projetos de prevenção precoce de doenças crônicodegenerativas. Esses projetos abrangem obesidade, doenças respiratórias, distúrbios comportamentais, repercussões mórbidas da restrição do crescimento intra-uterino e avaliação do impacto de doenças crônicas iniciadas na infância. Para que esses objetivos sejam atingidos é crucial a obtenção de marcadores genômicos de proteção ou susceptibilidade às doenças estudadas, uma área típica da Genômica Pediátrica.

Em linhas gerais, os principais requisitos para a efetiva aplicação clínica da genômica em Pediatria envolvem: 1) plataformas de alto rendimento para detecção de polimorfismos genéticos e estudo da expressão gênica em larga escala; 2) repositórios de material biológico articulados com bancos de informações clínicas e populacionais e com serviços ambulatoriais e hospitalares; 3 ) programas de ensino e treinamento em genômica clínica para estudantes e profissionais da saúde.

O Departamento de Pediatria da FMUSP atua nessas três áreas: o Laboratório de Genômica Pediátrica abriga as plataformas para estudo da expressão gênica, o Laboratório de Investigação Médica em Pediatria Clínica (LIM-36) do Instituto da Criança do HC-FMUSP ( $\mathrm{ICr}$ ) opera o banco de material biológico e esses dois laboratórios dão suporte ao Programa de Investigação em Genômica Pediátrica e aos programas de ensino e treinamento em Genômica Clínica para graduação, residência e pós-graduação senso estrito, como descrito adiante.

\section{A genômica na prática clínica}

Mudanças nos padrões de morbi-mortalidade na faixa etária pediátrica no Brasil mostram o continuado declínio da desnutrição, gastroenterites e doenças infecciosas. Por outro lado, surgem como problemas graves a obesidade e as síndromes metabólicas, o sedentarismo e os efeitos da degradação ambiental e social sobre a saúde de crianças e adolescentes. Cerca de $15 \%$ das crianças atendidas em consultas pediátricas apresentam algum distúrbio de comportamento, principalmente déficit de atenção e hiperatividade, além de ansiedade e depressão. A asma brônquica tem sido diagnosticada em cerca 
de $13 \%$ das crianças em idade escolar na cidade de São Paulo. Esse quadro está levando a um aumento exponencial dos casos de doenças crônicas com origem na infância, principalmente doenças cérebro e cárdio-vasculares ${ }^{7}$ ), diabetes ${ }^{8}$, doenças respiratórias $^{9} \mathrm{e}$ distúrbios de comportamento ${ }^{10}$. Em todas essas condições, estão sendo identificados fatores genéticos que, conjuntamente com fatores ambientais, determinam a susceptibilidade individual ao desenvolvimento da doença. A prevenção dessas doenças passa, então, a ter peso crescente na prática pediátrica e nos serviços de atenção à saúde de forma geral. Para isso, a identificação dos fatores de risco, ambientais e genéticos, e de seu mecanismo de interação é crucial para que se faça uma prevenção precoce e eficaz.

Considerando a importância da prevenção da doença crônica com origem na infância, alguns dos principais hospitais pediátricos norte-americanos, como Children's Hospital Bostone Children's Hospital of Philadelphia, estabeleceram centros dedicados à genômica clínica. Essas instituições perceberam acertadamente que, após a conclusão do Projeto Genoma Humano, estava aberto o caminho para se desvendar a interação gene-ambiente e identificar os componentes genéticos e ambientais nas doenças multifatoriais. Esses programas têm operado em duas linhas principais: i) estudos de polimorfismos de DNA e de variações estruturais no genoma para identificar indivíduos com risco aumentado de desenvolver determinada doença ${ }^{11}$; ii) estudos de expressão gênica para esclarecer o mecanismo molecular das doenças e para a obtenção de marcadores genômicos de diagnóstico, prognóstico e avaliação da resposta a agentes terapêuticos ${ }^{12}$.

Avanços na pesquisa genômica e seu impacto em pediatria

Variações estruturais no genoma e doenças complexas - Alterações submicroscópicas, conhecidas como copy number variants, ou CNVs, têm sido crescentemente associadas ao mecanismo causador de diversas doenças genéticas e degenerativas ${ }^{13} \mathrm{e}$, principalmente, à susceptibilidade a doenças complexas e à variabilidade individual na resposta a diversas drogas ${ }^{14,15}$. As CNVs interferem nos padrões de expressão gênica ${ }^{16}$ e estão associadas a patologias de interesse pediátrico, como distúrbios do comportamento ${ }^{17}$, susceptibilidade a infecções ${ }^{18}$, obesidade ${ }^{19}$ e asma ${ }^{20}$. A detecção e o estudo de CNVs em larga escala tornou-se possível com o desenvolvimento de microarrays de DNA para hibridização genômica comparativa (CGH), onde ganhos ou perdas de material genético podem ser identificados com precisão e em nível de resolução muito superior ao logrado com técnicas citogenéticas convencionais ${ }^{21}$.

Um ponto importante é que as associações entre CNVs e doenças dependem da estrutura genética das populações sob estudo e do seguimento dessas populações através do estudo de coortes. Não há estudos dessa natureza nas populações brasileiras, o que representa uma janela de oportunidade em pesquisa e, ao mesmo tempo, uma lacuna a ser preenchida. O Departamento de Pediatria da FMUSP, através do programa "Uma Nova Puericultura para Crianças que vão viver 100 Anos ou Mais" e do seguimento de coortes no Centro de Saúde Escola do Butantã, realizado no âmbito do Programa Região Oeste, tem vantagens comparativas para conduzir estudos sobre CNVs e doenças de interesse pediátrico no País.

Obtenção de biomarcadores para diagnóstico, prognóstico e identificação de alvos terapêuticos através do estudo da expressão gênica - O estudo diferencial da expressão gênica através de microarrays 22 é hoje um dos principais recursos para a investigação do mecanismo molecular das doenças (Hanegar). Essa tecnologia é também utilizada para o estabelecimento de associações entre padrões de expressão gênica com subtipos de doenças e resposta a agentes terapêuticos ${ }^{23}$. Esses padrões são chamados assinaturas moleculares. $\mathrm{Na}$ prática clínica, as assinaturas, depois de validadas por estudos funcionais e de seguimento, são substituídas por marcadores para alguns dos genes diferencialmente expressos, muitas vezes obtidos de forma simples e barata (imunoistoquímica, reação em cadeia da polimerase, etc.).

Marcadores genômicos diferenciais são extremamente úteis no diagnóstico, prognóstico e orientação terapêutica. Em oncologia, por exemplo, o uso de marcadores genômicos na prática clínica já é uma realidade em países desenvolvidos. É interessante notar que nesses países a mortalidade por câncer infantil declinou expressivamente nas últimas três décadas do século XX ${ }^{24}$. No Brasil, esse declínio tem sido menos acentuado, refletindo a desigualdade social e o acesso ainda limitado a recursos modernos de diagnóstico e tratamento ${ }^{25}$.

Epigenética - Epigenética é o estudo das alterações herdáveis no potencial de expressão gênica que não são causadas por mudanças na seqüência do $\mathrm{DNA}^{26}$. Essas alterações podem ser causadas por fatores ambientais e/ou endógenos, principalmente na vida intra-uterina e nos primeiros anos de vida ${ }^{27}$, sendo a modulação da atividade gênica mediada por mecanismos moleculares bem conhecidos: metilação do DNA em sítios promotores 
da transcrição, acetilação/desacetilação de histonas e ligação de microRNAs ao término 3' complementar do RNA mensageiro ${ }^{28}$. A desregulação epigenética é causa de diversas doenças, entre as quais: câncer, doenças cardiovasculares, diabetes, obesidade e distúrbios neurocomportamentais ${ }^{29}$. Evidências recentes, obtidas por pesquisadores do Instituto Karolinska, em Estocolmo, mostram que a metilação do DNA (que reduz a atividade de transcrição gênica) é significativamente maior em crianças nascidas de parto cesáreo comparativamente às nascidas de parto normal ${ }^{30}$. Presentemente, não se sabe como a expressão gênica é afetada nessas crianças, mas o parto cesáreo tem sido associado a risco aumentado para leucemia, alergia e diabetes. No Brasil, onde a incidência do parto cesáreo assume proporções alarmantes, esse dado abre importante área de pesquisa clínica com evidentes implicações para políticas de saúde pública.

\section{Perspectivas}

A disciplina de Pediatria Clínica dispõe de infra-estrutura assistencial, de ensino e de pesquisa para aplicar a tecnologia genômica ao estudo das patologias de interesse pediátrico com prevalência crescente em nosso meio, destacando-se as numerosas patologias crônicas de caráter familiar, tais como distúrbios de crescimento e doenças metabólicas de caráter familiar, doenças respiratórias crônicas, imunodeficiências primárias, doenças autoimunes e doenças genéticas associadas ou não a malformações múltiplas.

$\mathrm{Na}$ área da disciplina de Pediatria Preventiva e Social, a Genômica Pediátrica será instrumento essencial para os estudos de coortes em desenvolvimento no Centro de Saúde Escola do Butantã, com objetivo de se identificar o perfil de risco para doenças cardiovasculares, síndromes metabólicas e doenças respiratórias. Isso constituirá a base para a proposta de uma nova puericultura centrada na intervenção precoce para prevenir doenças crônicas da vida adulta.

$\mathrm{Na}$ área da disciplina de Pediatria Neonatal, a Genômica Pediátrica dará suporte ao manejo das infecções neonatais, principal causa da mortalidade neonatal tardia. Exemplo disso é o uso de marcadores genômicos em sepse: polimorfismos em genes ligados à resposta imune natural têm papel relevante na evolução da resposta à terapia ${ }^{31}$.

No cenário de convergência tecnológica que caracteriza a Medicina moderna, é essencial a formação de equipes multidisciplinares capacitadas a atuar num ambiente de contínua inovação. A introdução da Genômica na prática pediátrica faz parte desse processo de formação. A partir de 2008, o Depto. de Pediatria introduziu conteúdos de Genômica Pediátrica na grade curricular do terceiro ano de graduação em Medicina. Em 2009, dois projetos temáticos da FAPESP concedidos a docentes do Departamento ajudaram a consolidar essa linha de investigação no LIM-36. Com o desenvolvimento do Programa de Investigação em Genômica Pediátrica, será possível, através da interação com Serviço de Genética Clínica do ICr, implantar o ensino de genômica clínica na pós-graduação e oferecer treinamento nessa área aos residentes e a especialistas de todo o país.

Moreira-Filho CA. Pediatric genomics investigation at FMUSP Department of Pediatrics. Rev Med (São Paulo). 2010 abr.-jun.;89(2):83-7.

\begin{abstract}
The susceptibility for developing chronic diseases in adult life, such as diabetes, cardiovascular diseases or behavioral disorders, depends in a large extension on the interplay between genetic and environmental factors in fetal life and early childhood. The investigation on how changes in pre and post natal environment modify gene function, leading to permanent metabolic alterations and disease susceptibility is nowadays the main target of pediatric genomics. The knowledge obtained in this area of medical research will render possible in a near future that specific intervention in early life stages may conduct to improvements in human health. This article reviews recent progresses in pediatric genomics and summarizes the activities of the FMUSP Department of Pediatrics in this area.
\end{abstract}

KEY WORDS: Pediatrics; Genomics.

\section{REFERÊNCIAS}

1. Swanson JM, Entringer S, Buss C, Wadhwa PD. Developmental origins of health and disease: environmental exposures. Semin Reprod Med. 2009;27(5):391-402.
2. Gluckman PD, Hanson MA. Living with the past: evolution, development, and patterns of disease. Science. 2004;305(5691):1733-6.

3. Waterland RA, Michels KB. Epigenetic epidemiology of the developmental origins hypothesis. Annu Rev Nutr. 
2007;27:363-88

4. Weissleder R. Molecular imaging in cancer. Science. 2006;312(5777):1168-71.

5. Sebat J. Major changes in our DNA lead to major changes in our thinking. Nat Genet. 2007;39(7):S3-5.

6. Cupples LA, Arruda HT, Benjamin EJ, D'Agostino RB Sr, Demissie S, DeStefano AL, et al. The Framingham Heart Study 100K SNP genome-wide association study resource: overview of 17 phenotype working group reports. BMC Med Genet. 2007;8(1):S1.

7. McMahan CA, Gidding SS, Viikari JS, Juonala M, Kähönen M, Hutri-Kähönen N, et al. Association of pathobiologic determinants of atherosclerosis in youth risk score and 15-year change in risk score with carotid artery intima-media thickness in young adults (from the Cardiovascular Risk in Young Finns Study). Am J Cardiol. 2007;100(7):1124-9.

8. Oren A, Vos LE, Uiterwaal CS, Gorissen WH, Grobbee $\mathrm{DE}$, Bots ML. Change in body mass index from adolescence to young adulthood and increased carotid intima-media thickness at 28 years of age: the atherosclerosis risk in young adults study. Int $\mathrm{J}$ Obes Relat Metab Disord. 2003;27(11):1383-90.

9. Bjerg A, Hedman L, Perzanowski MS, Platts-Mills T, Lundbäck B, Rönmark E. Family history of asthma and atopy: in-depth analyses of the impact on asthma and wheeze in 7- to 8-year-old children. Pediatrics. 2007;120(4):741-8.

10. Zhao X, Leotta A, Kustanovich V, Lajonchere C, Geschwind $\mathrm{DH}$, Law $\mathrm{K}$, et al. A unified genetic theory for sporadic and inherited autism. Proc Natl Acad Sci U S A. 2007;104(31):12831-6.

11. Cohen J. Genomics. DNA duplications and deletions help determine health. Science. 2007;317(5843):1315-7.

12. Dalton WS, Friend SH. Cancer biomarkers - an invitation to the table. Science. 2006;312(5777):1165-8.

13. Vissers LE, Veltman JA, van Kessel AG, Brunner HG. Identification of disease genes by whole genome $\mathrm{CGH}$ arrays. Hum Mol Genet. 2005;14(2):R215-23.

14. Pastinen $\mathrm{T}, \mathrm{Ge} B$, Hudson TJ. Influence of human genome polymorphism on gene expression. Hum Mol Genet. 2006;15:R9-16.

15. Freishtat RJ, Teach SJ. Understanding genomics: implications for the emergency medicine physician and the treatment of asthma. Pediatr Emerg Care. 2006;22(1):71-5.

16. Stranger BE, Forrest MS, Dunning M, Ingle CE, Beazley $\mathrm{C}$, Thorne N, et al. Relative impact of nucleotide and copy number variation on gene expression phenotypes. Science. 2007;315(5813):848-53.

17. Sebat J, Lakshmi B, Malhotra D, Troge J, LeseMartin C, Walsh T, et al. Strong association of de novo copy number mutations with autism. Science. 2007;316(5823):445-9.
18. Daly KA, Brown WM, Segade F, Bowden DW, Keats BJ, Lindgren BR, et al. Chronic and recurrent otitis media: a genome scan for susceptibility loci. Am J Hum Genet. 2004;75(6):988-97.

19. Newell A, Zlot A, Silvey K, Arail K. Addressing the obesity epidemic: a genomics perspective. Prev Chronic Dis. 2007;4(2):A31.

20. Madore AM, Tremblay K, Hudson TJ, Laprise C. Replication of an association between 17q21 SNPs and asthma in a French-Canadian familial collection. Hum Genet. 2008;123(1):93-5.

21. Shaffer LG, Kashork CD, Saleki R, Rorem E, Sundin K, Ballif BC, et al. Targeted genomic microarray analysis for identification of chromosome abnormalities in 1500 consecutive clinical cases. J Pediatr. 2006;149(1):98102.

22. Nevins JR, Potti A. Mining gene expression profiles: expression signatures as cancer phenotypes. Nat Rev Genet. 2007;8(8):601-9.

23. Brenton JD, Carey LA, Ahmed AA, Caldas C. Molecular classification and molecular forecasting of breast cancer: ready for clinical application? Clin Oncol. 2005;23(29):7350-60.

24. La Vecchia C, Levi F, Lucchini F, Lagiou P, Trichopoulos D, Negri E. Trends in childhood cancer mortality as indicators of the quality of medical care in the developed world. Cancer. 1998;83(10):2223-7.

25. Ribeiro KB, Lopes LF, de Camargo B. Trends in childhood leukemia mortality in Brazil and correlation with social inequalities. Cancer. 2007;110(8):1823-31.

26. Jaenisch $\mathrm{R}$, Bird A. Epigenetic regulation of gene expression: how the genome integrates intrinsic and environmental signals. Nat Genet. 2003;33:245-54.

27. Fowden AL, Giussani DA, Forhead AJ. Intrauterine programming of physiological systems: causes and consequences. Physiology (Bethesda). 2006;21:2937.

28. Gluckman PD, Hanson MA, Cooper C, Thornburg KL. Effect of in utero and early-life conditions on adult health and disease. N Engl J Med. 2008;359(1):61-73.

29. Waterland RA, Michels KB. Epigenetic epidemiology of the developmental origins hypothesis. Annu Rev Nutr. 2007;27:363-88.

30. Schlinzig T, Johansson S, Gunnar A, Ekström TJ, Norman M. Epigenetic modulation at birth - altered DNA-methylation in white blood cells after Caesarean section. Acta Paediatr. 2009;98(7):1096-9.

31. Harding D. Impact of common genetic variation on neonatal disease and outcome. Arch Dis Child Fetal Neonatal. 2007;92(5):F408-13.

Artigo recebido em: 17/06/2010

Artigo aceito em:17/06/2010 